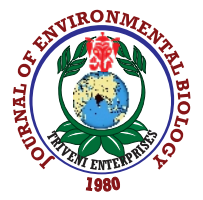

\title{
Identification, characterization and expression analysis of sucrose transporters in the model plant Nicotiana tabacum cv.petit havana
}

\author{
M. Abiramavalli and B. Usha* \\ Department of Genetic Engineering, SRM Institute of Science and Technology, Kattankulathur-603 203, India \\ *Corresponding Author Email : sundaram.usha@gmail.com
}

\begin{abstract}
Aim: Sucrose transporters (SUTs) are transmembrane proteins involved in transport of sucrose along the phloem pathway of plants. Nicotiana tabacum, a well-known model plant has been reported to have three sucrose transporters (NtSUT1, NtSUT3 and NtSUT4), till date. The present study was undertaken to identify new SUTs from its genome and characterize all the NtSUT genes of Nicotiana tabacum.
\end{abstract}

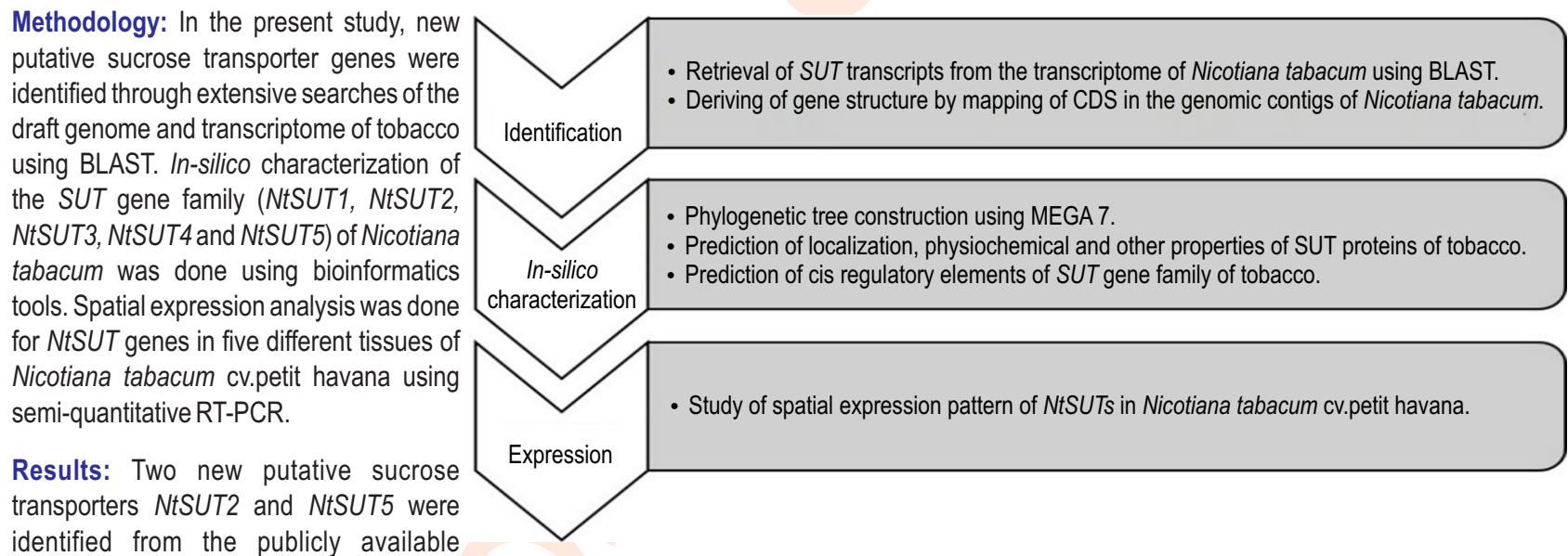

tobacco transcriptome data and characterized using in-silico tools. Multiple sequence alignment of deduced amino acids of all the five SUTs revealed the presence of highly conserved domains and signature histidine residue in the sugar binding motif. Phylogenetic analysis grouped NISUT1 and 3 into group 2, NtSUT4 and 5 into group 4 and NtSUT2 into group 3. NtSUT1 showed higher expression in leaves and flower while NtSUT2, 3 and 4 were abundant in root and flower. NtSUT5 showed a lower expression in all the tissues.

Interpretation: Based on the sequence information and structural similarities, it is clear that the two newly identified putative SUT genes (NISUT2 and NtSUT5) belonged to the SUT family of tobacco. This comprehensive study provides a consolidated data on characteristic features of SUT family proteins of tobacco.

Key words: Expression analysis, Nicotiana tabacum cv. petit havana, Phylogenetic classification, Sucrose transporters

How to cite : Abiramavalli, M. and B. Usha: Identification, characterization and expression analysis of sucrose transporters in the model plant Nicotiana tabacum cv.petithavana. J. Environ. Biol., 41, 803-811 (2020). 


\section{Introduction}

Sugars represent the main energy source in plants. Sucrose is the primary sugar transported from the source (leaves) to the sink (tissues) like young leaves, roots, flowers and seeds through phloem. This transport can be symplastic or apoplastic. Apoplastic transport is mediated by specific membrane proteins, called sucrose transporters (SUTs) which controls sucrose loading into the sieve element-companion cell complex (Zhang et al., 2019). Plant sucrose transporters belong to glycoside pentoside hexuronide (GPH) cation symporter family and a part of major facilitator superfamily. Almost all SUTs have been characterized as sucrose $/ \mathrm{H}^{+}$symporters, except a few facilitators (SUFs) which catalyse bidirectional sucrose transport in a $\mathrm{pH}$ and energy independent manner (Kühn and Grof, 2010). SUTs are highly hydrophobic proteins with 12 transmembrane helices possessing approximately 500-600 amino acids with an exception of few dicot members that contain an elongated central loop with approximately 60 additional amino acids. SUTs are either located in the plasma membrane of sieve elements or companion cells or in the vacuolar membrane. SUT family members, mainly involved in phloem loading are mostly localized in the plasma membrane whereas the vacuolar SUTs are reported to function as sucrose proton symporters by effluxing sucrose from the vacuoles (Hennion et al., 2019).

SUTs are classified into five groups based on their sequences and structural similarities. Group 1 consists of plasma membrane localized monocot SUTs which have moderate affinity for sucrose and catalyses the sucrose uptake into sink tissues. Group 2, being the largest and well-studied group, consists of plasma membrane localized dicot SUTs, which have high affinity for sucrose, involved in phloem loading and these were the first identified SUTs. Group 2 members have a wide range of substrate specificity than group 1. Group 3 consists of both monocots and dicots, most of the members are easily distinguishable from other groups with an extended cytoplasmic loop possessing 15-20\% more amino acids and they are proven to possess high affinity for sucrose. Also, studies suggest that some of the members of this group can act as potential sucrose sensors and not as symporters. Group 4 consists of both monocot and dicot SUTs with 10 fold lower affinity for sucrose than group 2. They transport a wide range of substrates but are more specific than group 2 . Some of the members under group 4 have been found to be localized on the vacuolar membrane and are involved in the efflux of sucrose. Group 5 is made exclusively of functionally uncharacterized monocot SUTs and they are different from group 1 (Braun and Slewinski, 2009). The only SUT which has been characterized from this group is OSSUT5, which has high affinity for sucrose and other substrates than group 1 and that explains the separation from group 1 (Sun et al., 2009).

Besides phloem loading, SUTs appears to influence various other processes such as pollen tube growth, retrieval of sucrose along the phloem pathway, flowering and photoperiod dependent induction of tuberization. Since SUTS are essential proteins in carbon partitioning, their transport mechanisms and metabolism are highly regulated at transcriptional and post transcriptional levels. SUT genes are proven to have distinct expression patterns during different developmental stages ( $\mathrm{Xu}$ et al., 2018). The spatio-temporal expression of many SUT genes of various plant species has already been well documented. For instance, in rice, OsSUT1 shows higher expression in germinating seeds, source leaf sheaths, panicles and developing grains and at lower levels in roots. OsSUT3 and OsSUT5 depicts highest expression in sink leaves and low expression in germinating seeds. OsSUT4 expresses only in sink leaves and OSSUT2 shows almost equal level of expression in all the tissues (Aoki et al., 2003). Similarly, in tomato, LeSUT4 expression was higher in young sink leaves, stems, cotyledons, and immature fruits. LeSUT1 was expressed more in source leaves with lower expression in all other organs. LeSUT2 expression was low in general but relatively higher in sink leaves and stems (Barker et al., 2000). In addition to organ and tissue specificity, the expression pattern of SUTs vary under nutrient deficit conditions, stress conditions, and at different developmental stages (Shiratake, 2007).

The availability of whole genome information of various plant species has led to the identification and characterization of SUTs in various plant species so far such as Oryza sativa, Solanum lycopersicum, Arabidopsis thaliana, and so on. (Sauer, 2007). So far, there are three SUT genes which are reported and characterized for the most widely used model plant and an important cash crop, Nicotiana tabacum (tobacco). However, only cDNA information, kinetic properties and expression data of NtSUT1, 3 and 4 are available (Bürkle et al., 1998; Lemoine et al., 1999; Okubo-Kurihara et al., 2011). Hence, the present study intended to identify other SUTs from the recently released whole genome information of Nicotiana tabacum and to analyse the gene structure, physio-chemical properties, presence of functionally important domains and conserved motifs, and presence of cis-acting elements in the upstream regions of all the NtSUT genes, besides documenting spatial expression pattern of NtSUTS in the cultivar Nicotiana tabacum cv. petit havana.

\section{Materials and Methods}

Identification of SUT genes in tobacco: Whole genome and transcriptome of three Nicotiana tabacum varieties (Basma Xanthi, TN 90 and K326) were carried out recently (Sierro et al., 2014). Transcriptome wide analysis was performed to identify sucrose transporter gene families using stand-alone version of BLASTn and BLASTp with more than $50 \%$ homology as cut off. NtSUT1, NTSUT3 and NtSUT4 CDNA sequences available in the NCBI database were used as query sequences. Amino acid sequences were deduced using NCBI ORF finder. Redundant sequences were removed after aligning the amino acid sequences using CLUSTAL Omega. The CDNA sequences of non-redundant proteins were used as queries to search for their appropriate contiguous gene sequences (contigs) from tobacco genome using BLASTn. Genomic contigs with lower e-values for 
SUT genes were retrieved and aligned manually with cDNA sequences for locating the introns. Schematic representation of exon-intron structure was obtained using Exon-Intron graphic maker tool.

In-silico analysis of NtSUT proteins: Physio-chemical properties of five sucrose transporter proteins including two new putative SUT proteins of tobacco identified in this study, were predicted using PROTPARAM (Gasteiger et al., 2005). Subcellular localization of these SUT proteins was analysed using CELLO server (Yu et al., 2006). Transmembrane helical domains of these proteins were predicted using OCTOPUS (Viklund and Elofsson, 2008). Amino acid sequences of SUTs of various plant species were aligned with SUTs of tobacco using tools CLUSTAL Omega and PRALINE (Bawono and Heringa, 2014) to ascertain the presence of highly conserved motifs among sugar transporters.

Phylogenetic analysis of NtSUTs: Amino acid sequences of sucrose transporters including the newly identified putative sucrose transporter sequences were aligned with those of different plant species using CLUSTAL Omega. Phylogenetic tree was constructed based on alignments using MEGA7 software (Kumar et al., 2016) by maximum likelihood method. To find the groups of identified NtSUTs, the tree was tested using bootstrap analysis with 1000 replicates.

Prediction of cis-regulatory elements using bioinformatics tools: Upstream $1500 \mathrm{bp}$ sequence of transcriptional start site of each gene were searched using PLANTCARE database to identify the cis-regulatory elements of NtSUT1-5 genes. Moreover, manual scanning was also done to identify the presence of sugar responsive elements such as sucrose box (NNAATCA) (Chen et al., 2002; Fillion et al., 1999) and pyrimidine box (CCTTTT, TTTTTTCC) (Washio, 2003).

Plant growth conditions and isolation of total RNA: N. tabacum cv. petit havana seeds (obtained from Tamil Nadu Agricultural University, Coimbatore, India) were thoroughly washed with sterile distilled water and surface sterilized with sodium hypochlorite $(2 \%)$. The seeds were sown in autoclaved soil and germinated in a plant growth Chamber at $28^{\circ} \mathrm{C}$ in $16 \mathrm{hr}$ light and $8 \mathrm{hr}$ dark cycle. Mature leaves, young leaves, stems, roots, and flowers harvested from 6 week-old-plants were cryopreserved. Total RNA was isolated from $100 \mathrm{mg}$ of all the tissues using TRIzol reagent (Thermo scientific, USA) following manufacturer's protocol. The samples were treated with DNase (NEB, England) and the purity and concentration was quantified using Nanodrop spectrophotometer (Thermo scientific, USA). Samples were checked on 1.5\% MOPS-formaldehyde gel and stored at $-80^{\circ} \mathrm{C}$.

Reverse transcription PCR (RT-PCR): From the quantified RNA samples, $1 \mu \mathrm{g}$ from each sample was reverse transcribed to cDNA using M-MuLV enzyme (NEB, England) and oligo dT primer. Enzyme, primers and RNA were incubated at $37^{\circ} \mathrm{C}$ for $50 \mathrm{~min}$ and the reaction was stopped at $70{ }^{\circ} \mathrm{C}$ for $15 \mathrm{~min}$. Second strand cDNA was synthesised using gene specific forward and reverse primers and the reaction was normalized with ubiquitin gene as endogenous control. PCR conditions were as follows: initial denaturation at $94{ }^{\circ} \mathrm{C}$, followed by 35 cycles for $30 \mathrm{sec}$ at $94^{\circ} \mathrm{C}, 30 \mathrm{sec}$ at 54 to $61^{\circ} \mathrm{C}$ (for different genes), $25 \mathrm{sec}$ at $72{ }^{\circ} \mathrm{C}$ and a final extension of 3 min at $72{ }^{\circ} \mathrm{C}$. The products were analysed on $1.5 \%$ agarose gel.

\section{Results and Discussion}

Tobacco is an allotetraploid and a model plant widely used for basic and genetic engineering research in plants. From the transcriptome of tobacco, 28 sequences homologous to NtSUTS were retrieved using the SUT sequences NtSUT1, NtSUT3 and NtSUT4 as queries. Of these, 2 non-redundant new sequences with mRNA IDs 116932 and 93499 were filtered. Based on the sequence homology with previously characterised SUTs of other plants, the two new putative SUT genes were named as NtSUT2 and NtSUT5. Deduced amino acid sequence of NtSUT2 showed $92 \%$ similarity with the well characterized LeSUT2 and StSUT2. And in the case of NtSUT5, it shared $81 \%$

Table 1: Characteristics of NtSUTS

\begin{tabular}{|c|c|c|c|c|c|c|c|c|c|}
\hline Gene name & Protein ID & Contig No. & $\begin{array}{l}\text { No. of } \\
\text { amino acids }\end{array}$ & $\begin{array}{l}\text { No. of } \\
\text { Exons }\end{array}$ & $\begin{array}{l}\text { No. of } \\
\text { introns }\end{array}$ & Mol. wt & pl & TMD* & Localization \\
\hline NtSUT1 & BAO47334 & AWOJ01093695 & 507 & 5 & 4 & $54 \mathrm{kDa}$ & 9.34 & 12 & $\begin{array}{l}\text { Plasma } \\
\text { membrane }\end{array}$ \\
\hline NtSUT2 & XP_016433446.1 & AYMY01357085 & 618 & 14 & 13 & $66 \mathrm{kDa}$ & 6.94 & 12 & $\begin{array}{l}\text { Plasma } \\
\text { membrane }\end{array}$ \\
\hline NtSUT3 & AAD34610 & AYMY01226069 & 521 & 6 & 5 & $56 \mathrm{kDa}$ & 9.10 & 12 & $\begin{array}{l}\text { Plasma } \\
\text { membrane }\end{array}$ \\
\hline NtSUT4 & BAI60050 & AWOK1098820 & 501 & 6 & 5 & $54 \mathrm{kDa}$ & 8.75 & 12 & $\begin{array}{l}\text { Plasma } \\
\text { membrane }\end{array}$ \\
\hline NtSUT5 & XP_016438053.1 & AWOJ01044985 & 491 & 4 & 3 & $54 \mathrm{kDa}$ & 8.70 & 11 & $\begin{array}{l}\text { Plasma } \\
\text { Plasma }\end{array}$ \\
\hline
\end{tabular}

*TMD-Transmembrane domain; *Mol.wt - Molecular weight; " 
Table 2: Classification of plant SUTs into groups

\begin{tabular}{|c|c|c|c|c|c|c|c|}
\hline \multirow[b]{2}{*}{ Name of the plant } & \multirow[b]{2}{*}{ No.of.SUTs } & \multicolumn{6}{|c|}{ Sub groups of plant SUTS } \\
\hline & & Group 1 & Group 2 & Group 3 & Group 4 & Group 5 & References \\
\hline Nicotiana tabacum & 5 & - & $\begin{array}{l}\text { NtSUT3, } \\
\text { NtSUT1 } \\
\text { AtSUC1, AtSUC2, }\end{array}$ & NtSUT2 & $\begin{array}{l}\text { NtSUT4, } \\
\text { NtSUT5 }\end{array}$ & - & $\begin{array}{l}\text { Sauer, } 2007 \\
\text { Leach et al., } 2017\end{array}$ \\
\hline Arabidopsis thaliana & 9 & - & $\begin{array}{l}\text { AtSUC5, AtSUC6, } \\
\text { AtSUC7, } \\
\text { AtSUC8, } \\
\text { AtSUC9 }\end{array}$ & AtSUC3 & AtSUC4 & - & Sauer, 2007 \\
\hline Oryza sativa & 5 & $\begin{array}{l}\text { OsSUT } \\
\text { OsSUT }\end{array}$ & - & OsSUT4 & OsSUT2 & OsSUT5 & Aoki et al., 2003 \\
\hline Zea mays & 3 & ZmSUT1 & - & ZmSUT2 & ZmSUT4 & - & Braun and Slewinski, 2009 \\
\hline
\end{tabular}

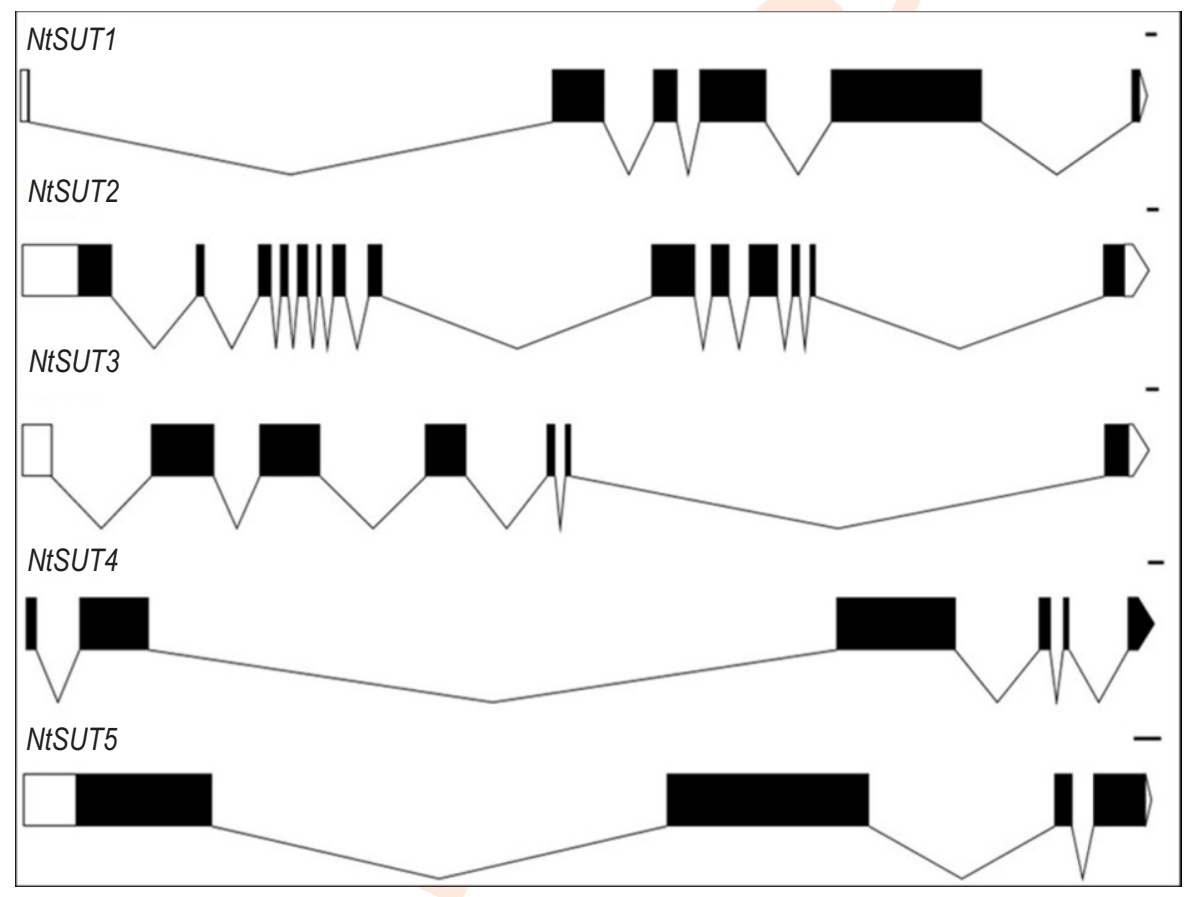

Fig. 1: Exon/intron structure of NtSUT genes. Exons are shown in black boxes and introns are represented with grey lines.

similarity with LeSUT4, $71 \%$ with AtSUC4 and $81 \%$ with NtSUT4, which has been already reported as group 4 transporters of tobacco (Leach et al., 2017). However, there were few variations in the number of amino acids and transmembrane domains between NISUT4 and NtSUT5, which proclaims that NtSUT5 could be a second member of the same group. Hence, to avoid confusion regarding nomenclature and to maintain consistency, it was designated as NtSUT5. Thus, NtSUT1-5 formed the sucrose transporter gene family in tobacco. The percentage similarities among the NtSUTs are predicted in Table 1. So far, the gene structural information of NtSUT1, 3 and 4 genes has not been studied in detail. Thus in this study, alignment of all the five cDNA sequences with their respective genomic contigs were done, which revealed the number and position of introns, exons, start and stop codons. There were 5 introns in NtSUT3 and NtSUT4, 4 introns in NtSUT1, 3 introns in NtSUT5 and 13 introns in NtSUT2 (Fig. 1). Promoter analysis of five SUTs revealed the presence of sugar responsive element, A-box (TACGTA), which is involved in regulation of sucrose transporters upon addition of sucrose (Kühn, 2011; Osuna et al., 2007). Sugar responsive elements such as sucrose box (NNAATCA) (Chen et al., 2002; Fillion et al., 1999) important for sugar responsive gene expression, pyrimidine box (CCTTTT) (Washio, 2003) partially involved in sugar repression were also observed. 


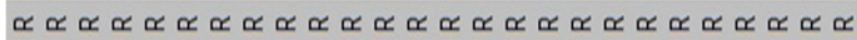

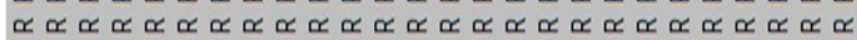
$\Upsilon \propto \alpha \propto \alpha \propto \alpha \propto \alpha \propto \alpha \propto \alpha \alpha \alpha \propto \alpha \propto \alpha \alpha \alpha \propto \alpha \propto \alpha \propto$

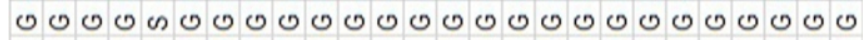

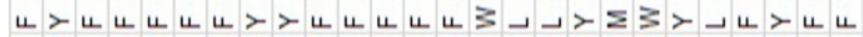

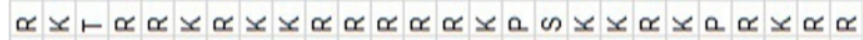

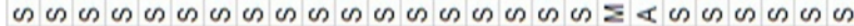

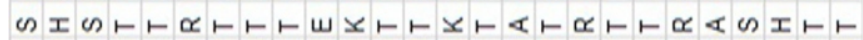

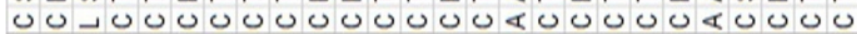
, , , , , , , , , , , , , , , , , , ., , , , , , , , , \&, , , , , , .

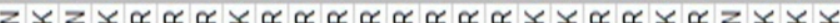
00000000000000000000000000

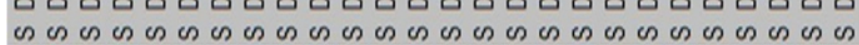

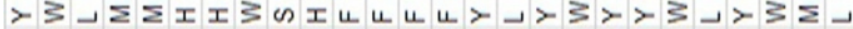

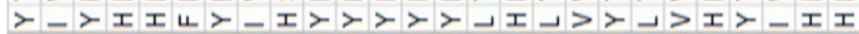
00000000000000000000000000

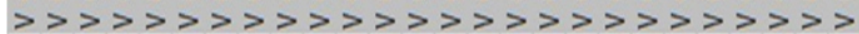

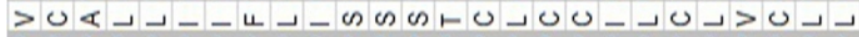

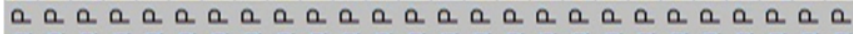
00000000000000000000000000

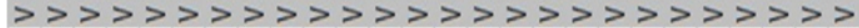

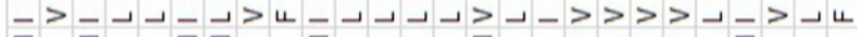

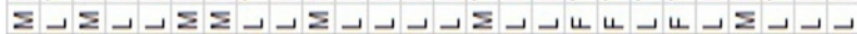

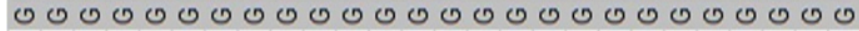

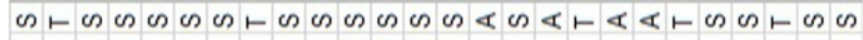
$---\lrcorner->--\lrcorner->>>--------\lrcorner--\lrcorner>$ a a a a a a a a a a a a a a a a a a a

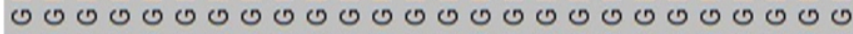
00000000000000000000000000

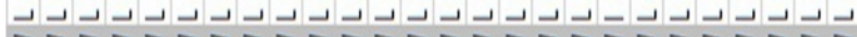

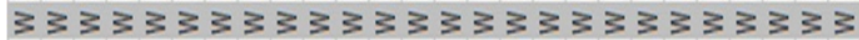

- - - - - - - - $\Sigma----\Sigma>\Sigma-1 \Sigma->---$ レ

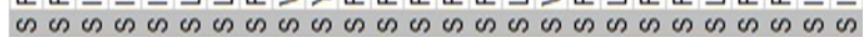

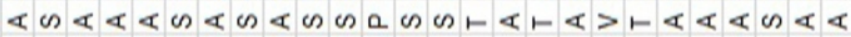

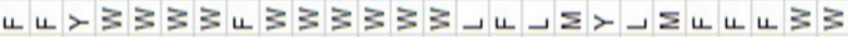

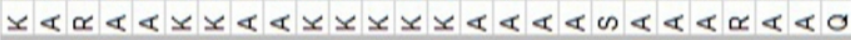
エエエエエエエエエエエエエエエエエエエエエエエエエエ

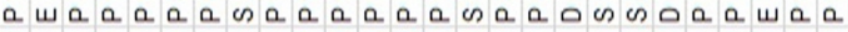
$---------->>>>-1----1-----$

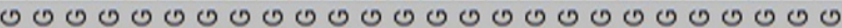

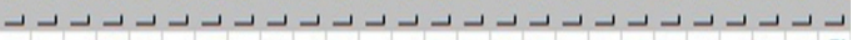

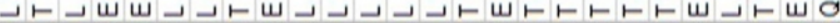

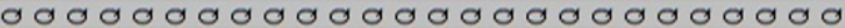
$>-\omega>>>>->->>>>>>>-\omega>->>->>$

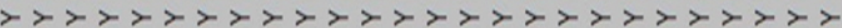
aca a a a a a a a a a a a a a a a a a

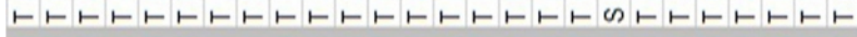
\lrcorner$-\lrcorner-\lrcorner-\lrcorner-\lrcorner-\lrcorner-\lrcorner-\lrcorner-\lrcorner,-\lrcorner-\lrcorner-\lrcorner-\lrcorner$

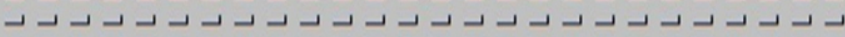
のののののののののののののののののののののののののの

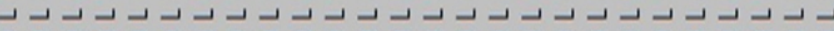

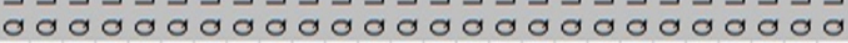

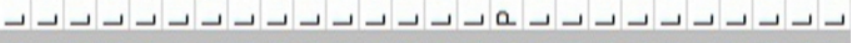

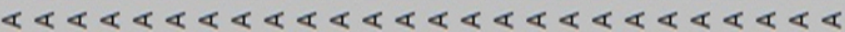

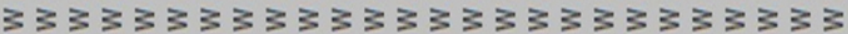
00000000000000000000000000

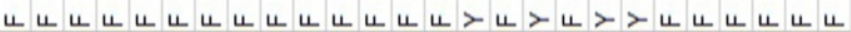
00000000000000000000000000 $>>>-->>>->---->>>>->>>>>-1$

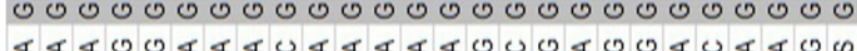

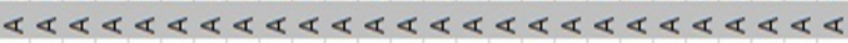
$->->>-->>----->>>>>>>>->>>$ のトのののののトのののののの

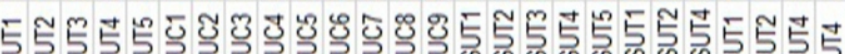

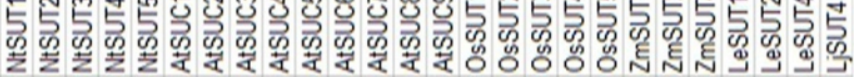


Besides, various cis-acting elements associated with important physiological processes such as light responsiveness (ACE, Box I, Spl, and Box 4), stress responsiveness (MBS, HSE, LTR, TC-rich repeats (ATTCTCTAAC), and EIRE) hormone responsiveness (GARE, ABRE, ARE, TCA and Me-JA motif), tissue specific expression (GCN4 motif, CAT, as-2-box, Skn-1-motif), and metabolism (Ibraheem et al., 2010) were also observed. These results propound that these sucrose transporter proteins might be playing role in regulating the photoperiodic control during flowering, regulating biotic and abiotic stresses.

Furthermore, to ascertain that the two newly identified putative sequences NtSUT2 and NtSUT5 belong to the SUT family of tobacco, they should have the characteristic features of plant SUT proteins. Also, previous studies have reported only the cDNA sequences for NtSUT1, NtSUT3 and NtSUT4 genes while their basic protein characteristics and their homology with other plant SUTs were not highlighted (Bürkle et al., 1998; Lemoine et al., 1999; Okubo-Kurihara et al., 2011). Hence, in this study, a detailed prediction of the characteristic features of all five NtSUT proteins has been well documented using bioinformatics tools and tabulated in Table 1. NtSUT2 is the largest of all SUTs (618 aa) and possess additional amino acids in its $\mathrm{N}$-terminus and central cytoplasmic loop. It carries 40 amino acid extension at the $\mathrm{N}$-terminus and an extended central cytoplasmic loop with insertion of 52 additional amino acids between transmembrane helices VI and VII, a feature distinct for group 3 transporters (AtSUC3, LeSUT2, and PmSUC3) (Sauer, 2007). NtSUT4 and NtSUT5 have shorter C-terminus, which is a proven feature of the members of group 4 sucrose transporters that usually have the shortest polypeptide sequences when compared to other family members (Sauer, 2007). The isoelectric point of all NtSUT proteins ranged from 8.70-9.30, except NtSUT2 which was only 6.94 , again a typical characteristic of group 3 transporters. All five proteins were predicted to be localized in the plasma membrane with 11 or 12 transmembrane domains. NtSUT1, NtSUT2, NtSUT3 and NtSUT4 possessed 12 transmembrane helical domains (TMD) whereas NtSUT5 possessed only 11 transmembrane helical domains (Table 1).

Multiple sequence alignment of the amino acid sequences of NtSUTs with other known functional plant SUTs depicted the presence of consensus sequence pattern derived from the highly conserved region of plant SUTS: QFGWALQLSLLTPY (TMD I); IWLCGP (TMD II); RXGRRR (between TMD II and TMD III) domains (Fig. 2). Amino acid sequence alignment also revealed the presence of highly conserved histidine residue (His-65), a signature for the identification of plant sucrose transporters (Lu and Bush, 1998). Based on their sequence information, structure, function and other biochemical properties, SUT proteins of various plant species can be classified into 5 different groups (Braun and Slewinski, 2009). A phylogenetic classification was done for 35 SUT proteins including tobacco using their amino acid sequences. A rooted phylogenetic tree was constructed by

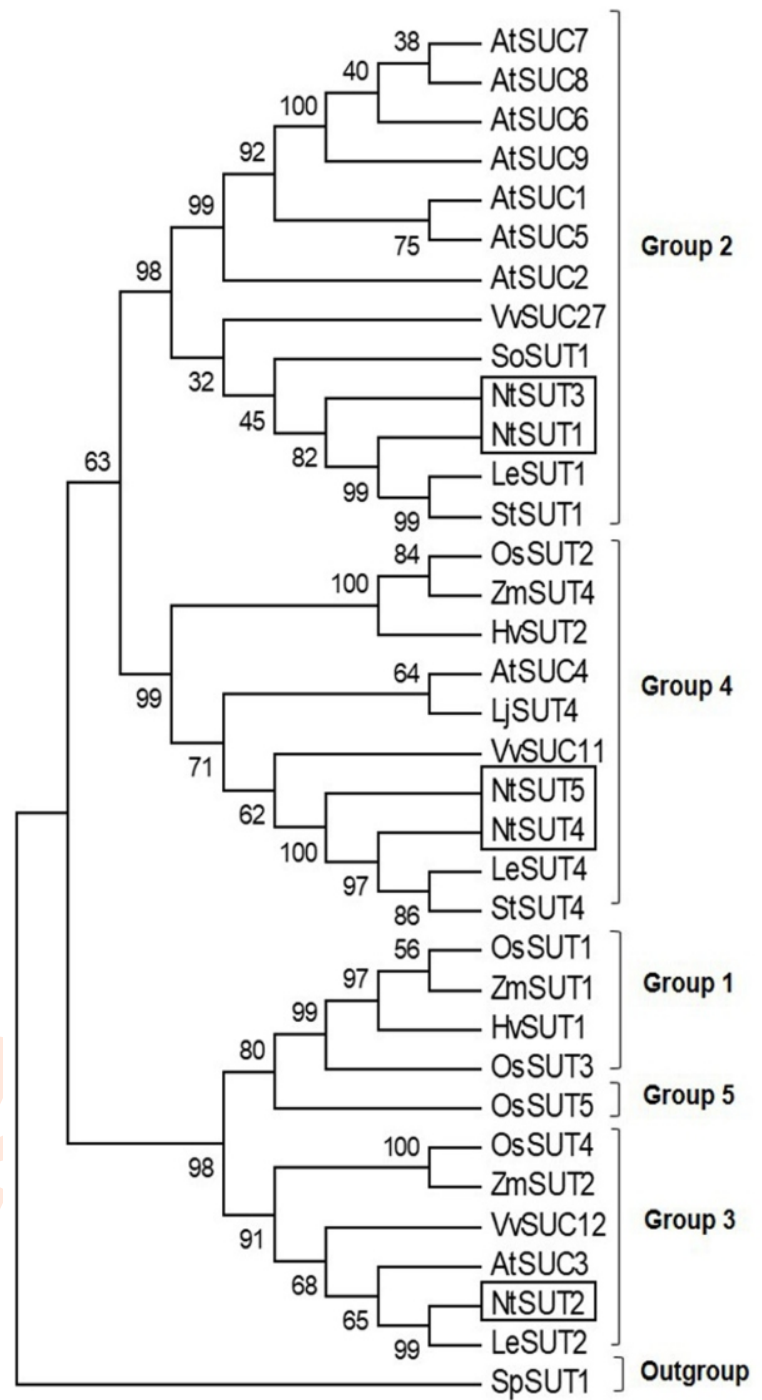

Fig. 3: Phylogenetic analysis of sucrose transporter family in tobacco. Maximum likelihood tree was generated by MEGA7 software with the amino acid sequences aligned using CLUSTAL omega. Following are the sequences used for the construction of phylogenetic tree: NtSUT1 (X82276.1), NtSUT3 (AF149981.1), NtSUT4 (BAI60050), NtSUT2 (XP_016433446.1), NtSUT5 (XP_016438053.1); Arabidopsis thaliana AtSUC1 (At1g71880), AtSUC2 (At1g22710), AtSUC3 (At2g02860), AtSUC4 (At1g09960), AtSUC5 (At1g71890), AtSUC6 (At5g43610), AtSUC7 (At1g66570), AtSUC8 (At2g14670), AtSUC9 (At5g06170); Oryza sativa OsSUT1 (Os03g0170900), OsSUT2 (Os12g0641400), OsSUT3 (Os10g0404500), OsSUT4 (Os12g44380), OsSUT5 (Os02g0576600); Vitis vinifera VvSUC11/VvSUT1 (HQ323256), VvSUC12 (HQ323257), VvSUT27 (HQ323258), Solanum tuberosum StSUT1 (CAA48915), StSUT4 (AAG25923.2); Lycopersicum esculentum LeSUT1 (X82275), LeSUT2 (AF166498); LeSUT4 (AF176950); Lotus japonicas, LjSUT4 (CAD61275); Hordeum vulgare HvSUT1 (CAB75882), HvSUT2 (CAB75881); Zea mays, ZmSUT1 (BAA83501), ZmSUT2 (AAS91375), ZmSUT4 (AAT51689); Spinacea oleracea SoSUT1 (Q03411); Schizosaccharomyces pombe, SpSUT1 was included as an outgroup. 


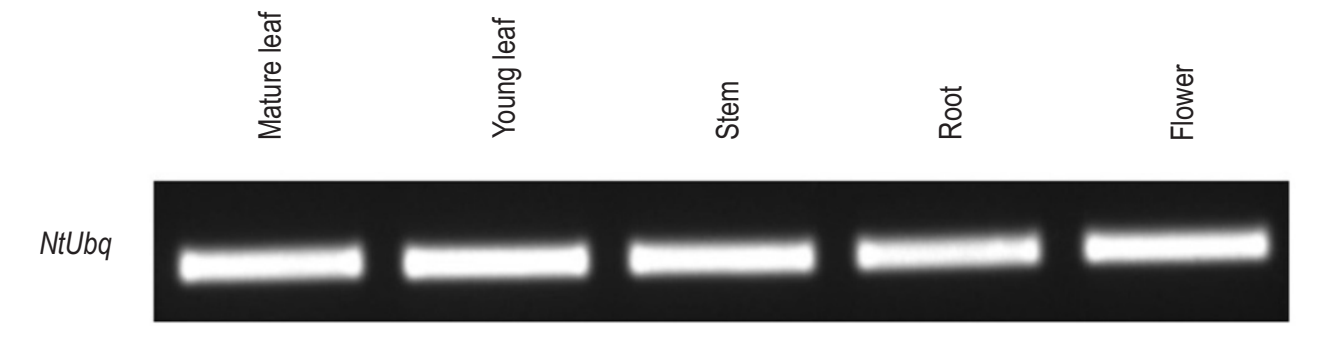

NtSUT1

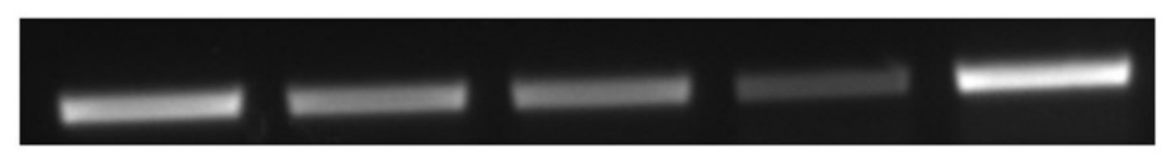

NtSUT2

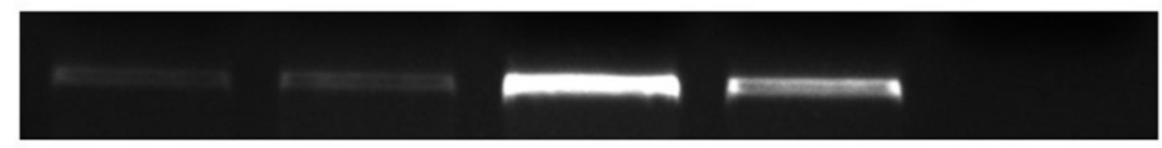

NtSUT3

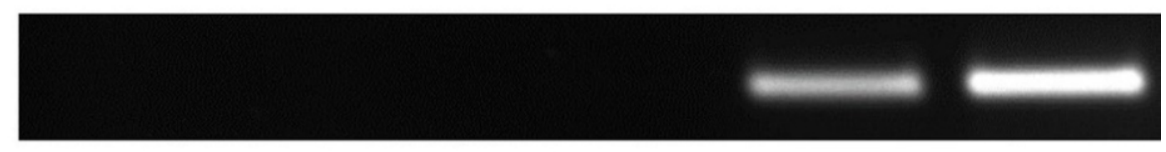

NtSUT4

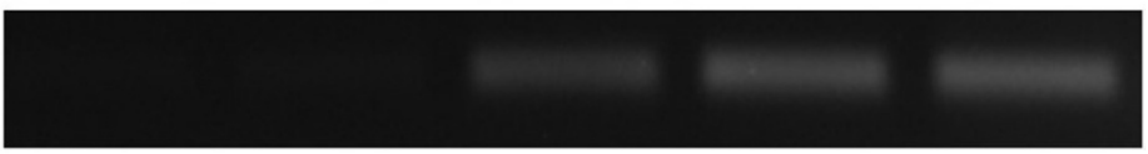

NtSUT5

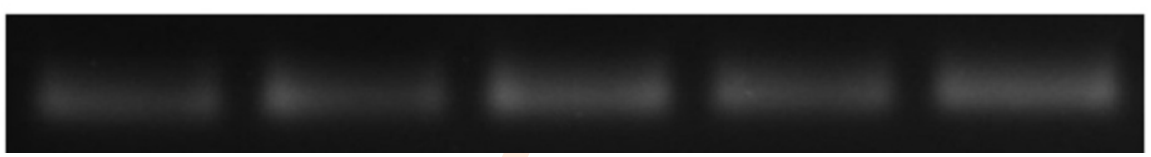

Fig. 4: Expression profile of NtSUTs in tissues of Nicotiana tabacum cv.petit havana. $1 \mu \mathrm{g}$ of DNase treated total RNA, isolated from mature and young leaves, stem, root and flower of tobacco plants were used for cDNA synthesis. Expression pattern was analysed using semi-quantitative reverse transcription PCR. Ubiquitin was used as endogenous control.

maximum likelihood method: bootstrap values with 1000 replicates using MEGA7.0 (Kumar et al., 2016) to examine the phylogenetic relationship between the sucrose transporters of tobacco and various plant species (Fig. 3). As earlier reports on phylogenetic analysis of plant sucrose transporters suggests, the three previously reported SUTs of tobacco belong to 2 different groups: group 2 and group 4. NtSUT1 fall under group 2 transporters as it is closely related to LeSUT1 by sharing $78 \%$ identity. NtSUT3 is a second member of same group by sharing $67 \%$ sequence identity with NISUT1. Similarly, NtSUT4 fall into group 4 transporters depicting close relationship with LeSUT4. On these terms, among the two newly identified NtSUT genes in this study, NISUT5 becomes a second member of group 4 transporter by sharing $81 \%$ identity with LeSUT4 and NtSUT4 whereas NtSUT2 falls into group 3 transporters, showing close relationship with LeSUT2 by sharing $88 \%$ sequence identity.
These results depicts that the SUT gene family ( 5 genes) of tobacco belong to 3 different groups (Table 2).

Expression of plant sucrose transporters is proven to be differentially and developmentally regulated. In this study, the spatial expression pattern of NISUT transcripts has been recorded using semi-quantitative reverse transcription PCR (RTPCR) (Fig. 4). Expression of NtSUT1 was high in the shoot tissues and flowers as compared to root. The transcript level was comparatively high in the source leaf tissues than stem. This observation is in concordance with the previous report that the expression of NISUT1 was low in the sink tissues and high in the source tissues (Bürkle et al., 1998). However, in the present study, NtSUT1 expression was high in the flower, which is a sink organ. Higher expression of SUT1 in the sink tissues is attributed to its functions such as retrieval of sucrose along the translocation 
and phloem unloading (Barker et al., 2000). SUT2 and SUT4 type transporters are proven to be expressed high in sink leaves and stems than source leaves (Barker et al., 2000). In a similar fashion, NISUT2 also showed high expression in the stem and root, reduced expression in the leaf tissues and showed no expression in the flower. Similarly, in the case of NtSUT4, the expression was moderate in the sink tissues such as stem and roots while there was no expression in the mature source leaves and young leaves which is concurrent with the previous reports on SUT4 of various plant species (Weise et al., 2000). However, in case of NISUT3, which has already been proved as a pollen specific transporter (Lemoine et al., 1999) shows high expression in the flower and a comparatively lower expression in the root tissue of Nicotiana tabacum cv.petit havana cultivar. There is hardly any expression seen in other tissues such as leaves and stem. However, the exact role of NtSUT3 in the roots is yet to be elucidated. NTSUT5 showed similar low level expression in all the tissues suggesting a role in sucrose uptake in all the tissues.

The amino acid sequence composition, sequence similarities, structural similarities, phylogenetic analysis, conserved domains and motifs of the two newly identified transporters (NtSUT2 and NISUT5) highly recommends them as putative sucrose transporter like proteins of tobacco. The present study, thus, propounds that there are five genes which comprises the SUT gene family in Nicotiana tabacum.

\section{Acknowledgments}

Authors wish to thank Ms. Deepika Manoharan, Junior Research Fellow, M.S. Swaminathan Research Foundation, Taramani, Chennai, for her technical assistance. This work was supported by a grant recieved from the Department of Science and Technology (DST)-SERB scheme for young scientists (SB/FT/LS-137/2012).

\section{References}

Aoki, N., T. Hirose, G.N. Scofield, P.R. Whitfeld and T.R. Furbank: The sucrose transporter gene family in rice. Plant Cell Physiol., 44, 223-232 (2003).

Barker, L., C. Kühn, A. Weise, A. Schulz, C. Gebhardt, B. Hirner, H. Hellmann, W. Schulze, J.M. Ward and W.B. Frommer: SUT2, a putative sucrose sensor in sieve elements. Plant Cell, 12, 1153-1164 (2000).

Bawono, P. and J. Heringa: PRALINE: A versatile multiple sequence alignment toolkit. Methods Mol. Biol., 1079, 245-262 (2014).

Braun, D.M. and T.L. Slewinski: Genetic control of carbon partitioning in grasses: Roles of sucrose transporters and tie-dyed loci in phloem loading. Plant Physiol., 149, 71-81 (2009).

Bürkle, L., J.M. Hibberd, W.P. Quick, C. Kühn, B. Hirner and W.B. Frommer: The $\mathrm{H}^{+}$-sucrose cotransporter NtSUT1 is essential for sugar export from tobacco leaves. Plant Physiol., 118, 59-68 (1998).

Chen, W., N.J. Provart, J. Glazebrook, F. Katagiri, H.S. Chang, T. Eulgem, F. Mauch, S. Luan, G. Zou and S.A. Whitham: Expression profile matrix of Arabidopsis transcription factor genes suggests their putative functions in response to environmental stresses. Plant Cell, 14, 559-574 (2002).
Fillion, L., A. Ageorges, S. Picaud, T.P. Coutos, R. Lemoine, C. Romieu and S. Delrot: Cloning and expression of a hexose transporter gene expressed during the ripening of grape berry. Plant Physiol,, 120, 1083-1094 (1999).

Gasteiger, E., C. Hoogland, A. Gattiker, S. Duvaud, M.R. Wilkins, R.D. Appel and A. Bairoch: Protein identification and analysis tools on the ExPASy server. In: The Proteomics Protocols Handbook (Ed.: J.M. Walker). USA., pp. 571-607 (2005)

Hennion, N., M. Durand, C. Vriet, J. Doidy, L. Maurousset, R. Lemoine and N. Pourtau: Sugars en route to the roots. Transport, metabolism and storage within plant roots and towards microorganisms of the rhizosphere. Physiol. Plant., 165, 44-57 (2019).

Ibraheem, O., C.E.J. Botha and G. Bradley: In-silico analysis of cisacting regulatory elements in $5^{\prime}$ regulatory regions of sucrose transporter gene families in rice (Oryza sativa Japonica) and Arabidopsis thaliana. Comput. Biol. Chem., 34, 268-283 (2010).

Kühn, C.: Sucrose transporters and plant development. In: Transporters and Pumps in Plant Signaling (Eds.: M. Geisler and K. Venema). Springer, Berlin, Heidelberg, pp. 225-251 (2011)

Kühn, C. and C.P.L. Grof: Sucrose transporters of higher plants. Curr. Opin. Plant Biol., 13, 287-297 (2010).

Kumar, S., G. Stecher and K. Tamura: MEGA7: Molecular Evolutionary Genetics Analysis version 7.0 for bigger datasets. Mol. Biol. Evol., 33, 1870-1874 (2016).

Leach, K.A., T.M. Tran, T.L. Slewinski, R.B. Meeley and D.M. Braun: Sucrose transporter 2 contributes to maize growth, development, and crop yield. J. Integr. Plant Biol., 59, 390-408 (2017).

Lemoine, R., L. Bürkle, L. Barker, S. Sakr, C. Kühn, M. Regnacq, C. Gaillard, C. Delrot and W.B. Formmer: Identification of a pollenspecific sucrose transporter-like protein NtSUT3 from tobacco. FEBS Lett., 454, 325-330 (1999).

Lu, J.M.Y. and D.R. Bush: His-65 in the proton-sucrose symporter is an essential amino acid whose modification with site-directed mutagenesis increases transport activity. Proc. Natl. Acad. Sci. India Sect. B Biol. Sci., 95, 9025-9030 (1998).

Okubo-Kurihara, E., T. Higaki, Y. Kurihara, N. Kutsuna, J. Yamaguchi and S. Hasezawa: Sucrose transporter NtSUT4 from tobacco BY-2 involved in plant cell shape during miniprotoplast culture. J. Plant Res., 124, 395-403 (2011).

Osuna, D., B. Usadel, R. Morcuende, Y. Gibon, O.E. Bläsing, M. Höhne, M. Gunter, B. Kamlage, R. Trethewey and W.R. Scheible: Temporal responses of transcripts, enzyme activities and metabolites after adding sucrose to carbon-deprived Arabidopsis seedlings. Plant J., 49, 463-491 (2007).

Sauer, N.: Molecular physiology of higher plant sucrose transporters. FEBS Lett., 581, 2309-2317 (2007).

Shiratake, K.: Genetics of sucrose transporter in plants. G3: Genes | Genomes | Genetics, 1, 73-80 (2007).

Sierro, N., J.N.D. Battey, S. Ouadi, N. Bakaher, L. Bovet, A. Willig, S. Geopfert, M.C. Peitsch and L.V. Ivanov: The tobacco genome sequence and its comparison with those of tomato and potato. Nat. Commun., 5, 3833 (2014).

Sun, Y., A. Reinders, K.R. LaFleur, T. Mori and J.M. Ward: Transport activity of rice sucrose transporters OsSUT1 and OsSUT5. Plant Cell Physiol., 51, 114-122 (2009).

Viklund, $H$. and A. Elofsson: OCTOPUS: improving topology prediction by two-track ANN-based preference scores and an extended topological grammar. Bioinformatics, 24, 1662-1668 (2008).

Washio, K.: Functional dissections between GAMYB and Dof transcription factors suggest a role for protein-protein associations in the gibberellin-mediated expression of the RAmy $1 \mathrm{~A}$ gene in the 
rice aleurone. Plant Physiol., 133, 850-863 (2003).

Weise, A., L. Barker, C. Kühn, S. Lalonde, H. Buschmann, W.B. Frommer and J.M. Ward: A new subfamily of sucrose transporters, SUT4, with low affinity/high capacity localized in enucleate sieve elements of plants. Plant Cell, 12, 1345-1355 (2000).

Xu, Q., S. Chen, R. Yunjuan, S. Chen and J. Liesche: Regulation of sucrose transporters and phloem loading in response to environmental cues. Plant Physiol., 176, 930-945 (2018).
Yu, C., Y. Chen, C. Lu and J. Hwang: Prediction of protein subcellular localization. Proteins Struct. Funct. Bioinforma., 64, 643-651 (2006).

Zhang, W., S. Wang, F. Yu, J. Tang, L. Yu, H. Wang and J. Li: Genomewide identification and expression profiling of sugar transporter protein (STP) family genes in cabbage (Brassica oleracea var. capitata L.) reveals their involvement in clubroot disease responses. Genes, 10,71 (2019). 